TITLE:

\title{
General method of solving the Schrodinger equation of atoms and molecules
}

AUTHOR(S):

Nakatsuji, $\mathrm{H}$

CITATION:

Nakatsuji, H. General method of solving the Schrodinger equation of atoms and molecules. PHYSICAL REVIEW A 2005, 72(6): 062110.

ISSUE DATE:

2005-12

URL:

http://hdl.handle.net/2433/39833

RIGHT:

Copyright 2005 American Physical Society 
PHYSICAL REVIEW A 72, 062110 (2005)

\title{
General method of solving the Schrödinger equation of atoms and molecules
}

\author{
Hiroshi Nakatsuji \\ Department of Synthetic Chemistry and Biological Chemistry, Graduate School of Engineering, Kyoto University, Katsura, Nishikyo-ku, \\ Kyoto 615-8510, Japan and Fukui Institute for Fundamental Chemistry, Kyoto University, 34-4 Takano-Nishihiraki-cho, Sakyo-ku, \\ Kyoto 606-8103, Japan
}

(Received 26 June 2005; published 13 December 2005)

\begin{abstract}
We propose a general method of solving the Schrödinger equation of atoms and molecules. We first construct the wave function having the exact structure, using the ICI (iterative configuration or complement interaction) method and then optimize the variables involved by the variational principle. Based on the scaled Schrödinger equation and related principles, we can avoid the singularity problem of atoms and molecules and formulate a general method of calculating the exact wave functions in an analytical expansion form. We choose initial function $\psi_{0}$ and scaling $g$ function, and then the ICI method automatically generates the wave function that has the exact structure by using the Hamiltonian of the system. The Hamiltonian contains all the information of the system. The free ICI method provides a flexible and variationally favorable procedure of constructing the exact wave function. We explain the computational procedure of the analytical ICI method routinely performed in our laboratory. Simple examples are given using hydrogen atom for the nuclear singularity case, the Hooke's atom for the electron singularity case, and the helium atom for both cases.
\end{abstract}

DOI: 10.1103/PhysRevA.72.062110

PACS number(s): 03.65.Ca, 03.65.Ge, 31.10.+z, 31.15.-p

\section{INTRODUCTION}

As noted by Dirac in 1929 [1], the Schrödinger equation (SE) provides a fundamental mathematical law for chemistry, though the importance of the relativistic theory is much larger now than then considered. The SE has quite a significant power of predicting chemical phenomena with very high accuracy. It also connotes physical and chemical laws that are conceptually useful for understanding chemical phenomena like the Fukui-Woodward-Hoffman rule [2,3], etc. So establishing a general method of solving the SE as accurately as possible is one of the ultimate purposes of theoretical chemistry. The scientific and practical merits brought about by this method are huge. First, we can make accurate predictions of chemical and related phenomena. This is a new evolution of quantum chemistry since it has long been a qualitative or semiquantitative science and could not do very accurate predictions except for very simple systems. Second, we can get chemical concepts as accurately as possible and can get even very fine understanding of chemistry. Third, it gives sound starting points of approximate theories. Since one has exact wave functions, one can make up approximate theories by neglecting some complex but minor terms. Finally, it even helps to predict a new phenomenon. When accurate theory and accurate experiment contradict severely, something must be wrong or missing in either of them and this may become a clue for a discovery of a new science. Such a situation could have never been expected before, since our theories could not be sufficiently accurate.

Despite its importance, however, there was no general method of solving the SE. Only one practical method of calculating the "exact" wave function was the full-CI method $[4,5]$. But the number of variables involved in the full $\mathrm{CI}$ is astronomical: even for moderate-size molecules with moderate-quality basis sets, the numbers of the variables are huge and cannot be handled even with modern computers. Therefore, applications of the full CI have been very limited.
Furthermore, even if we could get the full-CI solutions, they are actually far from the true solutions of the SE, because the basis sets used there are usually far from complete. It is necessary to develop a general theory for calculating the exact wave function in an analytical form.

In our series of papers [6-13], we have studied the structure of the exact wave functions, aiming to develop a general method of calculating the exact wave function. By exact wave function, we mean the solution of the SE. We have posed two questions: (1) What mathematical structure the exact wave function has? (2) Is a general theory possible that constructs the exact wave function systematically? Recently, we could have been able to accomplish this aim and the results were summarized in the previous communication [13]. We have formulated a general systematic method of solving the SE of atoms and molecules using the ICI (iterative configuration or complement interaction) formalism in an analytical expansion form up to a desired accuracy. A purpose of this paper is to formulate this method in a more general and detailed context.

The exact wave function $\psi$ is the solution of the SE,

$$
H \psi=E \psi,
$$

where the Hamiltonian $H$ is given by

$$
\begin{aligned}
H= & \sum_{i}-\frac{1}{2} \Delta_{i}-\sum_{i} \sum_{A} Z_{A} / r_{A i}+\sum_{i>j} 1 / r_{i j}=K+V=K+V_{n e} \\
& +V_{e e} .
\end{aligned}
$$

In our studies for formulating a general theory of solving the SE [6-13], two stages were experienced. The first stage was to clarify the structure of the exact wave function and to formulate a general theory to construct the exact wave function [6-11]. In the SE, the Hamiltonian determines the exact wave function, so that the exact wave function may be ex- 
pressed as a functional of the Hamiltonian, $f(H)$, applied to some appropriate function $\psi_{0}$, say,

$$
\psi=f(H) \psi_{0} .
$$

We have investigated some possible forms of the function $f(H)$, based on the combined use of the variational principle and the H-square theorem, and shown that the ICI and the SECC (simplest extreme coupled cluster) methods give the exact wave function in the sense of Eq. (3) above [6-8]. The general ECC method, called ECCND method (ND stands for the number of division of the Hamiltonian), was not guaranteed to be exact. A special example of ECCND was the CCGSD (coupled cluster general singles and doubles) method considered by Nooijen [14] and the present author [6] almost at the same time. Nooijen conjectured that it may describe the exact wave function with only GSD number of variables. The present author also started his study with a similar optimism, but reached a negative conclusion that the CCGSD cannot be exact and, instead, he proposed the ICIGSD method as a method to reach the exact wave function with essentially the GSD number of variables [6]. Later, the present author relaxed his opinion to that the CCGSD is not guaranteed to be exact: though the CCGSD cannot be guaranteed to be exact, it may happen to be exact because of its highly nonlinear character with respect to the GSD variables [8]. Later on, several studies have been reported about the exactness of the CCGSD [15-20]: in particular, Mukherjee and Kutzelnigg [20] have strengthened the present author's proof for the GSD case [6] by introducing the Leealgebraic concept, which is in the author's language, "generalized" Thouless transformation concept used in the argument for the coupled cluster singles (CCS) in Ref. [6].

We have shown that the full-CI solutions can actually be obtained with essentially single to GSD number of variables by performing the simplest ICI (SICI) and the ICIGSD calculations for some molecules in closed- and open-shell electronic structures $[10,11]$. We have shown a unique merit of the ICIGSD method that gave the full-CI solutions in a few iterations [11]. However, even so, the full-CI solutions are usually far from the true solutions of the SE, because of the incompleteness of the basis sets. So we wanted to solve the SE in an analytical form.

A serious problem had occurred in the second stage in constructing a general theory for solving the SE of atoms and molecules in an analytical form. That was the singularity problem $[12,13]$ caused by the singularity of the Coulomb potential $V$ involved in the Hamiltonian given by Eq. (2). The nuclear attraction term $V_{n e}$ and the electron repulsion term $V_{e e}$ diverge when $r_{A i}$ and $r_{i j}$, respectively, become zero. For these singularities, the integrals of the higher powers of the Hamiltonian diverge and so we cannot go further. We had to overcome this difficulty for developing the general theory of solving the SE.

We have proposed two ways to overcome this difficulty. One was done by introducing the inverse Schrödinger equation (ISE) [12] and the other by introducing the scaled Schrödinger equation (SSE) [13]. Both the ISE and SSE are equivalent to the original SE. In the formulation based on the ISE, it was difficult to explicitly represent the inverse Hamil- tonian in a closed analytical form, though even the expanded form worked well [12]. On the other hand, the formulation based on the SSE was easy and rigorous and yet had no practical difficulties.

In the previous communication [13], we have also introduced the free ICI method. This method is very flexible and yet gives a solution variationally better than the ordinary fixed ICI method [6,7]. We have formulated the free ICI method based on the SSE as a general method of solving the SE and given very briefly the preliminary results of applications to hydrogen atom, helium atom, and hydrogen molecule. The results were very encouraging, showing a high potentiality of the proposed method.

The purpose of this paper is to explain in some details the general method of solving the SE in an analytical expansion form, which is currently performed in our laboratory. We explain the theoretical background, introduce the SSE and related principles, and give a general method of constructing the wave function that has an exact structure. A merit of the free ICI method is described in some detail. Then, the method is applied to the hydrogen atom that has nuclear singularity, to Hooke's atom that has electron singularity, and to the helium atom that has both nuclear and electron singularities.

In the subsequent papers, we will show more general applications of the proposed method to the hydrogen molecule [21] and to a few-electron atoms: helium, lithium, beryllium, and boron-i.e., from two- to five-electron atoms [22]. We will also apply the present method to Hooke's atom in a more general way than given in this paper. Further, an extension of the present formalism to the relativistic case for solving the relativistic Dirac and Dirac-Coulomb equations has successfully been formulated and will appear in a separate paper [23].

\section{FORMALISM OF THE METHOD OF SOLVING THE SCHRÖDINGER EQUATION}

\section{A. Basic theory}

The SE given by Eq. (1) defines the exact wave function. The variational principle given by

$$
\langle\psi|H-E| \delta \psi\rangle=0
$$

is equivalent to the SE when the variation of $\psi$ is arbitrary. Actually, when the variation is done within some limited functional space, it gives the best possible $\psi$ within that space. On the other hand, the $\mathrm{H}$-square equation given by

$$
\left\langle\psi\left|(H-E)^{2}\right| \psi\right\rangle=0
$$

is valid only for the exact $\psi$. If $\psi$ satisfies the $\mathrm{H}$-square equation, this $\psi$ is guaranteed to be exact. Comparing Eqs. (4) and (5), we can easily get the following theorem [7]: When a $\psi$ including only one variable $C$ satisfies

$$
\frac{d \psi}{d C}=(H-E) \psi,
$$

it has the structure of the exact wave function, because the variationally best $\psi$ satisfies the $\mathrm{H}$-square theorem and so is exact. 
Two simplest $\psi$ 's that have the structure of the exact wave function with only one variable are the simplest ICI (SICI) wave function [7] and the simplest extreme coupled cluster (SECC) wave function [8]. The SICI wave function is defined by the recursion formula

$$
\psi_{n+1}=\left[1+C_{n}\left(H-E_{n}\right)\right] \psi_{n},
$$

where $n$ stands for the iteration number. At convergence, the SICI wave function is guaranteed to become exact though it includes only one variable at each iteration. The SECC wave function is compactly written as

$$
\psi=\exp [C(H-E)] \psi_{0},
$$

which includes only one variable $C$. One can show, however, from a different argument that the optimal $C$ of the SECC would be minus infinite [24].

Within a given limited basis set, the SICI provides a method to calculate the "exact" full-CI solution by using only one variable, in contrast to a huge number of variables in the ordinary full CI. Actually, we have applied the SICI method to many molecules in closed- and open-shell electronic structures using minimal and double- $\zeta$ basis sets and shown that it smoothly converges to the exact full-CI solution [10]. Because each iteration step of the SICI is variational, it converges from above to the exact solution. However, since the number of the variable is so small-i.e., only 1-the convergence speed was slow: it took 30-70 iterations to get the energies correct to 8 decimal figures of the full-CI energies [10].

We can introduce a larger number of variables in the ICI formalism [6,7]. Dividing the Hamiltonian into $N_{D}$ pieces ( $N_{D}$ stands for the number of division),

$$
H=\sum_{I=1}^{N_{D}} H_{I}
$$

and introducing the partial energy $E_{I, n}=\left\langle\psi_{n}\left|H_{I}\right| \psi_{n}\right\rangle$, we can define the ICIND as

$$
\psi_{n+1}=\left[1+\sum_{I}^{N_{D}} C_{I, n}\left(H_{I}-E_{I, n}\right)\right] \psi_{n},
$$

which is also guaranteed to become exact at convergence. When we introduce the second-quantized Hamiltonian

$$
H=\sum_{p r} v_{p}^{r} a_{r}^{\dagger} a_{p}+\sum_{p q r s} w_{p q}^{r s} a_{r}^{\dagger} a_{s}^{\dagger} a_{q} a_{p}
$$

and divide it into singles and doubles pieces, we can define the ICIGSD as

$$
\psi_{n+1}=\left(1+\sum_{p r} c_{p}^{r} a_{r}^{\dagger} a_{p}+\sum_{p q r s} c_{p q}^{r s} a_{r}^{\dagger} a_{s}^{\dagger} a_{q} a_{p}\right) \psi_{n},
$$

which has the GSD number of variables $\left\{c_{p}^{r}, c_{p q}^{r s}\right\}$. This ICIGSD is also guaranteed to become exact (full CI) at convergence [6]. Actually, we have formulated the ICIGSD first [6], showing that the exact full-CI solution can be solved by using only the GSD number of variables that are much smaller than the huge number of variables in the ordinary full CI.
We have used the ICIGSD method to calculate the full-CI solutions of closed- and open-shell molecules using the minimal and double- $\zeta$ basis sets [11]. We have shown that the convergence speed of the ICIGSD was much faster than that of the SICI: typically only 3-9 iterations were enough in contrast to 30-70 iterations in the SICI. This was essentially due to an increase in the number of variables from one to the GSD number. Further, we have discussed a special merit of the ICIGSD among the general ICIND.

Thus, with the ICI formalism, we can formulate a method of calculating the exact full-CI solution using only one to GSD number of variables at each iteration. However, as stated in the Introduction, the full-CI solution is usually far from the true solution of the SE. To get a true solution of the $\mathrm{SE}$, we have to formulate a method of solving the $\mathrm{SE}$ in an analytical form.

When we consider a method of analytically solving the $\mathrm{SE}$, we soon encounter a big obstacle, which is the singularity problem caused by the Coulomb potential included in the Hamiltonian. As explained in the Introduction, the second and third terms of the Hamiltonian given by Eq. (2) have the so-called nuclear and electron singularities, respectively. In the SE given by Eq. (1), the right-hand side (RHS) has no singularity and therefore the singularities caused by these terms must be canceled out completely within the lhs of the SE. For example, in the hydrogen atom, the nuclear attractive force must be canceled out by the centrifugal force produced by the kinetic motion of an electron and this cancellation occurs only for the exact wave function. Generally, from such considerations, we can formulate the nuclear and electron cusp conditions of the exact wave functions, which were first derived by Kato [25]. The exact wave function satisfies these conditions and therefore no singularities are left in the SE of Eq. (1).

However, for approximate wave functions, such complete cancellation does not occur and some serious problems occur. For example, we show in Table I the integrals of $H^{n}$ for the hydrogen atom over the exact and approximate wave functions (Stater type and Gaussian type). The exact wave function always gives the correct value, but the approximate one gives divergence for $n \geqslant 3$. This divergence is due to the nuclear singularity. Table II shows the integrals of the higher powers of the electron-electron repulsion operator, $\left(1 / r_{12}\right)^{n}$, over the approximate Slater-type and Gaussian-type helium wave functions with the optimized orbital exponents. Again, the integrals of $\left(1 / r_{12}\right)^{n}$ diverge for $n \geqslant 3$ for both types of orbitals. This divergence is due to the electron singularity. It is easy to show that such divergence is general for the integrals of the higher powers of Hamiltonian over approximate wave functions. An intuitive explanation of this divergence is as follows. When you make $n$ large for $(1 / r)^{n}$, it becomes like a well of an infinite depth, so that any integrals of this potential over approximate wave functions of atoms and molecules must diverge.

These results imply that the theory involving higher powers of Hamiltonian has difficulties when applied to atoms and molecules: numerical calculations become impossible when the basic integrals involved become infinite. The SICI secular equation involves the integrals of third power of Hamiltonian [7] and the SECC wave function involves higher pow- 
TABLE I. Integrals of the higher powers of the Hamiltonian and the scaled Hamiltonian of the hydrogen atom over the Slater-type and Gaussian-type orbitals.

\begin{tabular}{|c|c|c|c|}
\hline \multirow{2}{*}{$\begin{array}{l}\text { Operator } Q \text { in }^{\text {a }} \\
\langle\psi|Q| \psi\rangle /\langle\psi \mid \psi\rangle\end{array}$} & \multicolumn{2}{|c|}{ Slater-type } & \multirow{2}{*}{$\begin{array}{c}\text { Gaussian-type } \\
\alpha=0.3\end{array}$} \\
\hline & Exact $(\alpha=1.0)$ & Approximate $(\alpha=0.8)$ & \\
\hline$H$ & -0.5 & -0.48 & -0.4240387 \\
\hline$H^{2}$ & 0.25 & 0.4608 & 0.4886535 \\
\hline$H^{3}$ & -0.125 & $-\infty$ & $-\infty$ \\
\hline$v H v$ & $-\infty$ & $-\infty$ & $-\infty$ \\
\hline$H^{4}$ & 0.0625 & $\infty$ & $\infty$ \\
\hline$H g(g H)^{2}$ & -0.5625 & -0.624 & -0.1402396 \\
\hline $\mathrm{HgHgH}$ & -0.25 & -0.2624 & -0.0711785 \\
\hline$H g H^{2}$ & -0.1875 & -0.24576 & -0.5396678 \\
\hline$H g H^{3}$ & 0.09375 & $\infty$ & $\infty$ \\
\hline
\end{tabular}

${ }^{\mathrm{a}} H=-1 / 2\left(d^{2} / d r^{2}\right)-1 / r(d / d r)-1 / r, v=-1 / r, g=r$.

ers of Hamiltonian [8]. Therefore, both SICI and SECC suffer singularity problems when they are applied straightforwardly. The $t$-expansion method [26], the connectedmoments method [27], and the surplus function method [28] are the methods that suffer similarly the singularity problem. We note that this singularity problem is inevitable as far as the exact wave function is expressed like Eq. (3) as a functional of the Hamiltonian applied to an approximate wave function: the Maclaulin expansion of the function $f$ of Eq. (3) with respect to $H$ always includes the higher powers of $H$, which causes the singularity problem.

This singularity problem cannot be circumvented by introducing the finite-nucleus model [29]. Further, the finiteness of the nucleus is from a different physical origin and the theory itself should be valid independent of the finiteness of the nucleus. We also note that the singularity problem was rather vague in the modern quantum-chemistry technology where everything is expanded with finite basis functions. There, when you have a Hamiltonian matrix $\mathbf{H}$ defined within the finite basis space, you can calculate $\mathbf{H}^{n}$ essentially to an arbitrary $n$, though this is impossible if the basis is complete, since then $\left(\mathbf{H}^{n}\right)_{i j}=\left\langle i\left|H^{n}\right| j\right\rangle$ and the integrals on the

TABLE II. Integrals of several operators over approximate helium wave functions.

\begin{tabular}{ccc}
\hline \hline & \multicolumn{2}{c}{ Wave function $^{\mathrm{a}}$} \\
\cline { 2 - 3 } Operator & $\begin{array}{c}\text { Slater-type } \\
\alpha_{\mathrm{opt}}=Z-5 / 16\end{array}$ & $\begin{array}{c}\text { Gaussian-type } \\
\beta_{\mathrm{opt}}=0.7670\end{array}$ \\
\hline$H$ & $-729 / 256$ & -2.301 \\
$-Z / r_{1}-Z / r_{2}+1 / r_{12}$ & $-729 / 128$ & -4.602 \\
$1 / r_{12}$ & $135 / 128$ & 0.988 \\
$\left(1 / r_{12}\right)^{2}$ & $243 / 128$ & 1.534 \\
$\left(1 / r_{12}\right)^{3}$ & $+\infty$ & $+\infty$ \\
$\left(1 / r_{12}\right)^{4}$ & $+\infty$ & $+\infty$ \\
\hline \hline
\end{tabular}

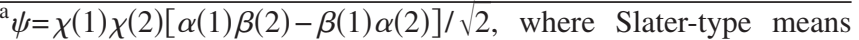
$\chi=\exp (-\alpha r)$ and Gaussian-type means $\chi=\exp \left(-\beta r^{2}\right)$.
RHS diverge when $n \geqslant 3$ as shown above. When the basis set is far from complete, the calculation may proceed without much difficulty, but it is simply due to the incompleteness of the basis set.

Now, how do we overcome this singularity problem? Otherwise, we cannot proceed further to find a way of calculating the exact wave functions of atoms and molecules. We have proposed two methods of solution: one was by introducing the inverse Schrödinger equation [12] and the other was by introducing the scaled Schrödinger equation [13]. In the case of the ISE, a problem was how to define the inverse Hamiltonian explicitly, though we have shown that even the expanded form of the inverse Hamiltonian was useful. On the other hand, in the case of the SSE reported in the previous communication, we could formulate a general straightforward method of calculating the exact wave function with avoiding the singularity problem. We will explain this method in some detail in the next section.

\section{B. Scaled Schrödinger equation and related theorems}

We define the scaled Schrödinger equation (SSE) by

$$
g(H-E) \psi=0,
$$

where the scaling function $g$ is a function of electron coordinates. It is a multiplicative operator and does not commute with the Hamiltonian. It is always positive (or always negative) except at the singular points. Even at the singular point $r_{0}$, the scaling function satisfies

$$
\lim _{r \rightarrow r_{0}} g V \neq 0,
$$

where $V$ is the potential operator in the Hamiltonian, in order not to eliminate the information of the Hamiltonian at the singular points: the singularity of the potential itself is also important information of the Hamiltonian, though it causes the singularity problem. Equation (14) means that $1 / g$ is a milder function than the potential itself in the limit of $r \rightarrow r_{0}$.

The SSE is equivalent to the original SE: When the SE holds, the SSE holds. Conversely, when the SSE holds, we 
multiply $1 / \mathrm{g}$ from the left of Eq. (13) and obtain the SE. This is valid even at the singular points because of Eq. (14).

There are many possibilities in the choice of the $g$ function. A choice is $g=1 /\left(-V_{n e}+V_{e e}\right)$, where $V_{n e}$ and $V_{e e}$ are the nuclear-electron attraction operator and electron-electron repulsion operator, respectively. A different choice is $g$ $=-1 / V_{n e} V_{e e}$ or more explicitly the inverse of the product of each Coulombic potential. However, this $g$ function is very complex and makes the ICI wave function very complex. Further, when one of the product terms in the $g$ function becomes zero, the other terms can be arbitrary, so that the restriction may not be complete. When we adopt the free ICI method described below, the freedom of the choice of the $g$ function becomes wider and one choice is $g=-1 / V_{n e}$ $+1 / V_{e e}$ or, more explicitly, the sum of all the inverses of the Coulomb potentials involved in the Hamiltonian. In this case, however, the ICI formulation also produces the functions that are singular, so that we have to eliminate such functions from our expansion bases of the free ICI wave function: the wave function must be integrably finite by its definition. Another choice is to take $g=r /(r+a)$ with $a$ being a constant for each Coulomb potential $1 / r$. This applies to both the product form and the sum form explained above. Actually, the functional form of the scaling function is very important in the ICI calculations. The choice of the $g$ function affects the easiness of integral evaluations and the convergence speed of the ICI calculations.

The SSE is very flexible as seen from its definition, and so it should be useful not only for atomic and molecular systems where the potential has Coulomb singularity, but also for more general cases where the singularity is steeper or less steeper than the Coulombic one.

Table I shows the integrals of the operators including both $g$ and $H$ for the case of hydrogen atom. For the potential $V$ $=-1 / r$, we have chosen the scaling function $g=r$. We see that the integrals of the higher powers of the scaled Hamiltonian, $(g H)^{n}$, exist for both exact and approximate wave functions even for $n \geqslant 3$, in contrast to the higher powers of the original Hamiltonian $H$. Note further that the integrals of the operators of the forms $H(g H)^{n}$ and $H^{2}(g H)^{n}$ also exist. These operators appear when we calculate the Hamiltonian matrix element and the matrix of the $H^{2}$ operator, respectively, over the ICI wave functions based on the SSE. So it is generally true that the integrals $g^{n} H^{m}$ over ordinary wave functions exist as long as $0 \leqslant m-n \leqslant 2$. Similarly, the integrals of the higher powers of the electron-electron repulsion operator, $\left(1 / r_{i j}\right)^{n}$, exists if $n \leqslant 2$. We will see later that these properties are very important in constructing the free ICI wave functions, as explained below, and calculating the exact wave functions of atoms and molecules. Note that $\left\langle\psi\left|g^{n}\right| \psi\right\rangle$ exists since $\psi$ is exponentially decaying function which decays much faster than the powers of $r$.

The variational principle given by Eq. (4) and the $\mathrm{H}$-square theorem given by Eq. (5) play very important roles in the formulation of our theory. We now show that the SSE also has the variational equation and the $\mathrm{H}$-square theorem as the original SE. This makes it possible to formulate the ICI theory based on the SSE, which leads to the ICI theory free from the singularity problem. Since the SE and the SSE are equivalent, this ICI theory also leads to the exact wave function.

The variational formula associated to the SSE is written from the SSE as

$$
\langle\delta \psi|g(H-E)| \psi\rangle=0 .
$$

For arbitrary $\delta \psi$, this equation is equivalent to the SSE. This equation may also be used to calculate the best possible wave function within the given functional form of $\psi$. The energy $E$ may be defined by

$$
E=\langle\psi|g H| \psi\rangle\langle\psi|g| \psi\rangle,
$$

which is called scaled energy and is different from the Ritz energy,

$$
E=\langle\psi|H| \psi\rangle /\langle\psi \mid \psi\rangle .
$$

The scaled energy does not have the upper-bound property, differently from the Ritz energy. For the exact wave function, both scaled and Ritz energies become equal to the exact energy.

The H-square theorem for the SSE is written as

$$
\langle\psi|(H-E) g \cdot g(H-E)| \psi\rangle=0,
$$

which is valid only for the exact wave function that satisfies the SSE or SE. The proof is very simple: this equation is rewritten as $\int|g(H-E) \psi|^{2} d \tau=0$, whose integrand is always positive or zero, and therefore, the integrand must be zero in order that the integral of them is zero, which leads to the SSE. The converse is self-evident. Q.E.D. Another important $\mathrm{H}$-square theorem of the SSE is

$$
\langle\psi|(H-E) g(H-E)| \psi\rangle=0,
$$

which is valid since $g$ is always positive or zero, so that we can define $\sqrt{g}$ and $\int|\sqrt{g}(H-E) \psi|^{2} d \tau=0$ is satisfied only when $\sqrt{g}(H-E) \psi=0$, which leads to the SE. Equation (19) is useful for showing the exactness of the ordinary (Ritz) variational solution of the SICI based on the SSE, as shown below.

We can formulate the SICI based on the SSE, just like that based on the SE. When a $\psi$ including only one variable $C$ satisfies

$$
\frac{d \psi}{d C}=g(H-E) \psi
$$

it has the structure of the exact wave function. Putting Eq. (20) into the variational formula given by Eq. (15), we obtain Eq. (18): the variationally best $\psi$ satisfies the H-square theorem and is therefore exact. In this formulation, we used the variational principle associated to the SSE, but we can also use the ordinary variational principle given by Eq. (4). When you put Eq. (20) into the ordinary variational principle given by Eq. (4), we obtain Eq. (19) which guarantees that the variational best is exact if $\psi$ has the structure of the exact wave function given by Eq. (20). This argument is important because when we have $\psi$ having the structure of the exact wave function as defined by Eq. (20), we can calculate its unknown variable $C$ either with the ordinary variational principle given by Eq. (4) or by the scaled variational principle 
given by Eq. (18). Of course, the ordinary variational principle is more useful, because it has the upper-bound nature (Ritz) but the scaled one does not.

Now we can formulate the simplest ICI theory based on the SSE. The SICI based on the SSE is defined by the recursion formula

$$
\psi_{n+1}=\left[1+C_{n} g\left(H-E_{n}\right)\right] \psi_{n} .
$$

It is different from the original one given by Eq. (7) simply by the existence of the $g$ function which works to eliminate the singularity problem. By differentiating $\psi_{n+1}$ with respect to the single variable $C_{n}$, we obtain an equation similar to Eq. (20) and this equation becomes identical to Eq. (20) at convergence where everything does not depend on the iteration number $n$. Thus, at convergence, the SICI based on the SSE is guaranteed to be exact.

The unknown variable $C_{n}$ of the SICI can be calculated in two different ways. One is to use the variational principle given by Eq. (15) and the other is to use the ordinary variational principle given by Eq. (4). From Eq. (15) we obtain two-dimensional secular equation

$$
\begin{aligned}
& \left\langle\psi_{n}\left|g\left(H-E_{n+1}\right)\right| \psi_{n}\right\rangle C_{0, n}+\left\langle\psi_{n}\right|\left(H-E_{n+1}\right) g \cdot g\left(H-E_{n}\right) \\
& \quad \times\left|\psi_{n}\right\rangle C_{1, n}=0, \\
& \left\langle\psi_{n}\left|\left(H-E_{n}\right) g \cdot g\left(H-E_{n+1}\right)\right| \psi_{n}\right\rangle C_{0, n}+\left\langle\psi_{n}\right|\left(H-E_{n}\right) g \cdot g(H \\
& \left.\quad-E_{n+1}\right) g\left(H-E_{n}\right)\left|\psi_{n}\right\rangle C_{1, n}=0,
\end{aligned}
$$

and from Eq. (4) a secular equation

$$
\begin{aligned}
& \left\langle\psi_{n}\left|\left(H-E_{n+1}\right)\right| \psi_{n}\right\rangle C_{0, n}+\left\langle\psi_{n}\left|\left(H-E_{n+1}\right) g\left(H-E_{n}\right)\right| \psi_{n}\right\rangle C_{1, n} \\
& \quad=0, \\
& \quad\left\langle\psi_{n}\left|\left(H-E_{n}\right) g\left(H-E_{n+1}\right)\right| \psi_{n}\right\rangle C_{0, n}+\left\langle\psi_{n}\right|\left(H-E_{n}\right) g(H \\
& \left.\quad \quad-E_{n+1}\right) g\left(H-E_{n}\right)\left|\psi_{n}\right\rangle C_{1, n}=0,
\end{aligned}
$$

where the coefficient $C_{n}$ of Eq. (21) is given by $C_{n}$ $=C_{1, n} / C_{0, n}$ : because of the normalization relation, we have only one variable. Referring to Table I, we see that all the integrals involved in Eqs. (22) and (23) exist. As described above, the solution of Eq. (23) satisfies the Ritz variational principle and is therefore more favorable in actual calculations than that of Eq. (22).

We can also formulate the simplest ECC theory based on the SSE as

$$
\psi=\exp [C g(H-E)] \psi_{0} .
$$

It is easy to show that this $\psi$ satisfies Eq. (20) and is therefore exact. It gives a compact expression of the exact wave function. Because of the existence of the $g$ function, this SECC is free from the singularity problem. It is known, however, that the coefficient $C$ is infinitely large for the exact ground state and this makes the usage of the SECC less popular. However, we have formulated the usage of the SECC wave function by expanding it in finite terms and the results of applications were encouraging [8]. In this paper our main concern is the ICI theory and therefore detailed discussions on the SECC wave functions based on the SSE are postponed to the future papers.
The H-square theorem based on the SSE can be generalized by introducing the partitioned Hamiltonian

$$
H=\sum_{I=1}^{N_{D}} H_{I}
$$

where $N_{D}$ stands for the number of division. The partitioning may be into kinetic, nuclear-attraction, and electronrepulsion operators of the Hamiltonian given by Eq. (2), where the nuclear attraction operator may be further divided into different nuclei or into the singles and doubles terms for the Hamiltonian given by Eq. (11). The H-square theorem for the partitioned Hamiltonian is given by a set of the $N_{D}$ equations,

$$
\left\langle\psi\left|(H-E) g \cdot g\left(H_{I}-E_{I}\right)\right| \psi\right\rangle=0,
$$

with $I=1, \ldots, N_{D}$. $E_{I}$ is defined by $\left\langle\psi\left|g\left(H_{I}-E_{I}\right)\right| \psi\right\rangle=0$. Similarly to Eq. (19), we also have another $\mathrm{H}$-square theorem

$$
\left\langle\psi\left|(H-E) g\left(H_{I}-E_{I}\right)\right| \psi\right\rangle=0,
$$

with $I=1, \ldots, N_{D}$ again, because we can define $\sqrt{g}$ from the definition of the $g$ function. We can show that when either of these partitioned H-square theorems holds for all the $N_{D}$ equations, this $\psi$ is exact. The proof is straightforward and so is not given here, except we note that we use the variational equation given by Eq. (15) for the proof of Eq. (26) and Eq. (4) for the proof of Eq. (27). You may refer to the proofs given in Refs. [6,7].

Based on the partitioned $\mathrm{H}$-square theorem, we can generalize the ICI theory so as to include the $N_{D}$ variables,

$$
\psi_{n+1}=\left[1+\sum_{I}^{N_{D}} C_{I, n} g\left(H_{I}-E_{I, n}\right)\right] \psi_{n},
$$

which was called ICIND method [7]. When this ICIND is calculated with either of the variational equations, Eqs. (15) and (4), it gives the exact wave function at convergence. In the proof, we use either combination, Eqs. (15) and (26) or Eqs. (4) and (27).

The calculation of the unknown variables $\left\{C_{I, n}\right\}$ in the ICIND wave function given by Eq. (28) is done either with the variational principle given by Eq. (15) or with the ordinary variational principle given by Eq. (4). The secular equation obtained with the variational principle given by Eq. (15) is written as

$$
\begin{gathered}
\left\langle\psi_{n}\left|g\left(H-E_{n+1}\right)\right| \psi_{n}\right\rangle C_{0, n}+\sum_{I}\left\langle\psi_{n}\right| g\left(H-E_{n+1}\right) \cdot g\left(H_{I}-E_{I, n}\right) \\
\times\left|\psi_{n}\right\rangle C_{I, n}=0 \\
\left\langle\psi_{n}\left|\left(H_{J}-E_{J, n}\right) g \cdot g\left(H-E_{n+1}\right)\right| \psi_{n}\right\rangle C_{0, n}+\sum_{I}\left\langle\psi_{n}\right|\left(H_{J}\right. \\
\left.\quad-E_{J, n}\right) g \cdot g\left(H-E_{n+1}\right) g\left(H_{I}-E_{I, n}\right)\left|\psi_{n}\right\rangle C_{I, n}=0
\end{gathered}
$$

and the secular equation derived from the ordinary variational principle given by Eq. (4) is written as 


$$
\begin{aligned}
& \left\langle\psi_{n}\left|\left(H-E_{n+1}\right)\right| \psi_{n}\right\rangle C_{0, n}+\sum_{I}\left\langle\psi_{n}\right|\left(H-E_{n+1}\right) \cdot g\left(H_{I}-E_{I, n}\right) \\
& \quad \times\left|\psi_{n}\right\rangle C_{I, n}=0, \\
& \left\langle\psi_{n}\left|\left(H_{J}-E_{J, n}\right) g\left(H-E_{n+1}\right)\right| \psi_{n}\right\rangle C_{0, n}+\sum_{I}\left\langle\psi_{n}\right|\left(H_{J}-E_{J, n}\right) \\
& \quad \times g\left(H-E_{n+1}\right) g\left(H_{I}-E_{I, n}\right)\left|\psi_{n}\right\rangle C_{I, n}=0,
\end{aligned}
$$

both for all $J\left(J=1, \ldots, N_{D}\right)$. The coefficient $C_{I, n}$ of Eq. (28) is related to the coefficients $C_{I, n}$ of Eq. (29) or (30) by $C_{I, n}$ $=C_{I, n} / C_{0, n}$. When we do not partition the Hamiltonian, Eqs. (29) and (30) reduce to Eqs. (22) and (23), respectively, of the SICI case. In actual calculations, Eq. (30) is more convenient, since the solution satisfies the upper-bound nature of the Ritz variational principle.

Here, we explain how we perform analytical calculations with the ICI method. We take the SICI method given by Eq. (21) for example. The initial function $\psi_{0}$ may be HartreeFock wave function, etc., which is anyway some analytical function. For analytical calculations, we use the Hamiltonian given by Eq. (2), which is the sum of the kinetic operator and the potential operators. The kinetic operator is a sum of the differentiation operators with respect to the electron coordinates and the differentiations are always possible for any kinds of analytical functions. There are many mathematical programs available that perform such differentiations easily, like MAPLE, MATHEMATICA, REDUCE, etc. The potential operators and the $g$ function are simply multiplicative functions. Thus the result of applying the SICI operator to $\psi_{0}$ is an analytical function with the unknown variable $C_{0}$. Repeating iterations, we always obtain the analytical functions with some unknown coefficients. In some general choice of the $g$ function, the resultant function may include the functions that have singularities causing the divergence of the integrals: such functions should be eliminated since they do not satisfy the integrability condition that the physical wave function must satisfy. Finally, the variable coefficients involved are calculated with the variational principle.

The ICI method we routinely use in our laboratory for calculating the analytical exact wave function is not the SICI or ICIND given above. These ICI methods are characterized by the following two features. (1) They generate the functions that converge iteratively to the exact wave function. (2) The number of the variables is small and fixed to one or $N_{D}$ throughout the calculations. In our routine calculations, we relax the second property. Namely, we collect all the independent functions $\left\{\phi_{k}\right\}^{(n)}, k=1, \ldots, M_{n}$, included in the $n$th iteration function of the SICI given by Eq. (21) and use them for expanding our wave function-namely,

$$
\psi_{n+1}=\sum_{k=1}^{M_{n}} c_{k, n} \phi_{k},
$$

where $M_{n}$ is the number of the independent functions included in $\left\{\phi_{k}\right\}^{(n)}$. The coefficients $\left\{c_{k, n}\right\}, k=1, \ldots, M_{n}$, are calculated with the variational principle (the ordinary Ritz variational principle is easier to use). Since the functions $\phi_{k}$ are generated with the scaled Hamiltonian, we no longer have the singularity problem. Even if we may have some singular functions due to a general use of the $g$ functions, we simply throw such functions out since they do not satisfy the physical condition ( $Q$ condition) for the wave function. We call this method the free ICI method. Since the ICI formulation guarantees to reach the exact wave function, the free ICI method gives the best possible wave function, in a variational sense, at each iteration. When $M_{n}$ is not so large, we need not to keep it to $N_{D}$. Further, in the free ICI method, no accumulation of errors occurs, since no variables of the earlier iteration step are used in the later iteration step. We can start $n$th iteration step without performing the earlier $n-1$ iterations. The free ICI method is very flexible. It makes many variants possible.

Thus, at sufficiently large $n$, we have the analytical function in the form of Eq. (31) that has the structure of the exact wave function: when the variables involved are determined by the variational principle, it gives the exact wave function. We can reach any accuracy by adjusting the iteration number $n$.

We have applied the ICI method, the free ICI method in particular, to atomic and molecular systems. In order to confirm the accuracy and the usefulness of the method, we have applied it to the hydrogen atom, helium atom, and hydrogen molecule, for which the exact or very accurate wave functions are already known. We have further applied it to manyelectron atoms: lithium, beryllium, and boron (three- to fiveelectron atoms) and $\mathrm{He}^{-\mathrm{H}^{+}}$, a two-electron molecule. We will summarize these calculations separately in the literatures $[21,22,30]$

In the present paper, we show the applications to the hydrogen atom, to the Hooke's atom, and to the helium atom. They are the examples of the systems including the nuclear singularity, the electron singularity, and the both, respectively.

\section{SIMPLE APPLICATIONS}

\section{A. Hydrogen atom}

The exact solution of the hydrogen atom is a classical subject of quantum chemistry. The Hamiltonian for the $\mathrm{s}$ state of the hydrogen atom is

$$
H=-\frac{1}{2} \frac{\partial^{2}}{\partial r^{2}}-\frac{1}{r} \frac{\partial}{\partial r}-\frac{Z}{r}
$$

and the exact wave function and energy are $\psi=\exp (-Z r)$ and $E=-0.5$ a.u. for $Z=1$. As an ICI problem, the hydrogen atom is an example of the nuclear singularity and therefore, even for this simplest example, the ICI approach fails without the introduction of the SSE and ISE. The solution with introducing the ISE was given previously [12], and here we give the solution with introducing the SSE.

As a simplest choice of the $g$ function, we choose $g=r$, which satisfies the condition given by Eq. (14). The initial function $\psi_{0}$ is chosen to be $\psi_{0}=\exp (-\alpha r)$ with $\alpha=1.5$. $\alpha$ $=1.0$ is the exact wave function. We perform three different ICI-namely, SICI $\left(N_{D}=1\right)$, ICIND $\left(N_{D}=3\right)$, and free ICI. In ICIND $\left(N_{D}=3\right)$, we have divided the Hamiltonian given by 
TABLE III. ICI calculation of hydrogen atom based on the SSE.

\begin{tabular}{|c|c|c|c|c|c|c|c|c|c|}
\hline \multirow[b]{2}{*}{$n$} & \multicolumn{2}{|c|}{ SICI (ND=1) } & \multirow[b]{2}{*}{$n$} & \multicolumn{2}{|c|}{ ICIND (ND=3) } & \multirow[b]{2}{*}{$n$} & \multirow[b]{2}{*}{$M$} & \multicolumn{2}{|c|}{ Free ICI } \\
\hline & $\begin{array}{c}\text { Ritz } \\
\text { energy }\end{array}$ & $\begin{array}{l}\text { Scaled } \\
\text { energy }\end{array}$ & & $\begin{array}{c}\text { Ritz } \\
\text { energy }\end{array}$ & $\begin{array}{l}\text { Scaled } \\
\text { energy }\end{array}$ & & & $\begin{array}{c}\text { Ritz } \\
\text { energy }\end{array}$ & $\begin{array}{l}\text { Scaled } \\
\text { energy }\end{array}$ \\
\hline 0 & -0.375 & -0.625 & 0 & -0.375 & -0.625 & 0 & 1 & -0.375 & -0.625 \\
\hline 1 & -0.375 & -0.375 & 1 & -0.375 & -0.375 & 1 & 2 & -0.491025404 & -0.512259526 \\
\hline 2 & -0.465594827 & -0.411923413 & 2 & -0.497053531 & -0.516537938 & 2 & 3 & -0.465594827 & -0.501470244 \\
\hline 3 & -0.481256589 & -0.434488719 & 3 & -0.499652721 & -0.494662663 & 3 & 4 & -0.499954132 & -0.500144830 \\
\hline 4 & -0.488165311 & -0.448981506 & 4 & -0.499974617 & -0.499580437 & 4 & 5 & -0.499997229 & -0.500011697 \\
\hline 5 & -0.492183091 & -0.458915958 & 5 & -0.499989900 & -0.499133267 & 5 & 6 & -0.499999844 & -0.500000825 \\
\hline 6 & -0.494625230 & -0.465959942 & 6 & -0.499998499 & -0.499699622 & 6 & 7 & -0.499999992 & -0.500000053 \\
\hline 7 & -0.496147453 & -0.471256973 & 7 & -0.499999309 & -0.499755042 & 7 & 8 & -0.500000000 & -0.500000003 \\
\hline 8 & -0.497154350 & -0.475303857 & 8 & -0.499999805 & -0.499859453 & 8 & 9 & & -0.500000000 \\
\hline 9 & -0.497839922 & -0.478500137 & 9 & -0.499999903 & -0.499897917 & & & & \\
\hline 10 & -0.498324376 & -0.481062407 & 10 & -0.499999961 & -0.499930983 & & & & \\
\hline 11 & -0.498674837 & -0.483160593 & 11 & -0.499999979 & -0.499948993 & & & & \\
\hline 12 & -0.498934961 & -0.484899446 & 12 & -0.499999990 & -0.499963105 & & & & \\
\hline 13 & -0.499131605 & -0.486362182 & 13 & -0.499999994 & -0.499971758 & & & & \\
\hline 14 & -0.499283108 & -0.487604403 & 14 & -0.499999997 & -0.499978750 & & & & \\
\hline 15 & -0.499401536 & -0.488671137 & 15 & -0.499999998 & -0.499983211 & & & & \\
\hline 16 & -0.499495476 & -0.489594209 & 16 & -0.499999999 & -0.499986999 & & & & \\
\hline 17 & -0.499570866 & -0.490399870 & 17 & -0.499999999 & -0.499989465 & & & & \\
\hline 18 & -0.499632078 & -0.491107471 & 18 & -0.500000000 & -0.499991650 & & & & \\
\hline 19 & -0.499682257 & -0.491733231 & 19 & & -0.499993097 & & & & \\
\hline 20 & -0.499723785 & -0.492289504 & 20 & & -0.499994420 & & & & \\
\hline 21 & -0.499758430 & -0.492786793 & & & & & & & \\
\hline 22 & -0.499787561 & -0.493233307 & & & & & & & \\
\hline 23 & -0.499812222 & -0.493636113 & & & & & & & \\
\hline 24 & -0.499833240 & -0.494000854 & & & & & & & \\
\hline 25 & -0.499851257 & -0.494332439 & & & & & & & \\
\hline 26 & -0.499866789 & -0.494634860 & & & & & & & \\
\hline 27 & -0.499880247 & -0.494911621 & & & & & & & \\
\hline 28 & -0.499891966 & -0.495165616 & & & & & & & \\
\hline 29 & -0.499902215 & -0.495399407 & & & & & & & \\
\hline 30 & -0.499911217 & -0.495615134 & & & & & & & \\
\hline
\end{tabular}

${ }^{a} n$ : iteration number. $M$ : number of independent functions.

Eq. (32) into three terms. The number of variables is unity in SICI, 3 in ICIND $\left(N_{D}=3\right)$, and increases as the iteration proceeds in the ICI. We calculated both Ritz energy and scaled energy given by Eqs. (16) and (17), respectively, and the results are given in Table III.

We expect from Table I that the singularity problem should not occur in the present method and actually, as seen from Table III, we get a smooth convergence to the exact energy in all cases, though the convergence rate is different among the different ICI methods and between the different definitions of the energy. For the SICI case, we have only one variable and, therefore, the convergence rate is very slow. When we use three variables in ICIND $\left(N_{D}=3\right)$, the convergence becomes much faster. When we use the free ICI method, the number of the variables increases as the iteration proceeds, one by one in this case, as the iteration number increases, and the convergence is fastest among the three ICI methods: the Ritz energy converges after seven iterations and the scaled energy by eight iterations to the exact energy up to nine decimal figures. Between the Ritz and scaled energies, the Ritz energy always converges faster and satisfies the upper-bound nature. The scaled energy does not necessarily satisfy the upper-bound nature: it may be even lower than the true energy.

The results of Table III indicate the usefulness of the free ICI method with the Ritz energy. The ICIND $\left(N_{D}=3\right)$ method may also be useful because in this case the number of the variables is always 3 or $N_{D}$.

Starting from the initial function $\psi_{0}=\exp (-1.5 r)$, the ICI method with $g=r$ improves the wave function order by order as the iteration proceeds. Actually, the wave function at $n$th iteration is written as $\psi_{n}=\exp (-1.5 r)\left(\sum_{i=0}^{n} c_{i} r^{i}\right)$. Since the ex- 
act wave function is $\exp (-1.0 r)$, the ICI correction term is nothing else but the variational approximation to the expansion, $\exp (0.5 r)=\sum_{i=0} c_{i} r^{i}$, with $c_{i}=0.5^{i} / n !$. The free ICI wave function at $n=7$ was $\left(c_{0}, c_{1}, c_{2}, c_{3}, c_{4}, c_{5}, c_{6}, c_{7}\right)=(1.000000$, $0.500262,0.124126,0.022132,0.001656,0.000621$, $-0.000049,0.000008)$. The sequence given by $c_{i}=0.5^{i} / n$ ! is $(1.000000,0.500000,0.125000,0.020833,0.000260$, $0.000022,0.000002)$. Since the energy is correct to nine decimal figures, the wave function would be correct roughly to four decimal figures, in accordance with the Eckert theorem. At higher iterations, we have actually confirmed that the first eight coefficients of the free ICI wave function agree exactly with the above numbers of the mathematical sequence. For general atomic and molecular systems, the exact wave functions cannot be written with single analytical functions, so that their best analytical expressions would be in an analytical expansion form as given in this example.

In comparison with the usage of the inverse SE reported in the previous paper [12], the calculation reported here is more straightforward. Though we could do analytical calculations based on the ISE for the hydrogen atom with the expanded inverse Hamiltonian and the results were rather satisfactory, it is difficult to generalize such method to manyelectron atoms and molecules, but the present method based on the SSE is straightforwardly applicable to any atoms and molecules.

\section{B. Hooke's atom}

Hooke's atom [31] is not a real atom, but an imaginative two-"electron" atom whose attractive potential is harmonic and the inter-"electron" repulsion is Coulombic; namely, its Hamiltonian is given by

$$
H=-\frac{1}{2}\left(\Delta_{1}+\Delta_{2}\right)+\frac{k}{2}\left(\left|\mathbf{r}_{1}\right|^{2}+\left|\mathbf{r}_{2}\right|^{2}\right)+\frac{1}{\left|\mathbf{r}_{1}-\mathbf{r}_{2}\right|^{2}} .
$$

The harmonic potential is nonsingular, but the Coulombic potential is singular [32]. We consider Hooke's atom whose Hooke's harmonic constant is $k=1 / 4$. A remarkable character of Hooke's atom is that its exact solution is known. For the above Hamiltonian, the exact solution is

$$
\psi=\exp \left[-\frac{1}{4}\left(r_{1}^{2}+r_{2}^{2}\right)\right]\left(1+\frac{r_{12}}{2}\right)
$$

and the exact energy is $E=2.0$ a.u.

We apply the ICI method to the above Hooke's atom. We take our initial guess as $\psi_{0}=\exp \left[-\frac{1}{4}\left(r_{1}^{2}+r_{2}^{2}\right)\right]$, an eigenfunction when the "electron" repulsion term in Eq. (33) is missing, and our $g$ factor as $g=r_{12}$ : the $g$ factor is necessary only for the electron repulsion operator. The free ICI result is summarized in Table IV. We see that the result converges to the exact solution at second iteration. There, both the energy and wave function become equal to the exact ones. Even if we perform further iterations, the correction is identically zero for both the energy and wave function, as it must be so. Thus the ICI method has given the exact solution of Hooke's atom.
TABLE IV. ICI energy and wave function for Hooke's twoelectron atom.

\begin{tabular}{cccc}
\hline \hline Iteration & Dimension & Energy (a.u.) & $\Delta \psi^{\mathrm{a}}$ \\
\hline 0 & 1 & 2.064189584 & 1 \\
1 & 2 & 2.0 & $+r_{12} / 2$ \\
2 & 2 & 2.0 & 0 \\
\hline
\end{tabular}

${ }^{\mathrm{a}} \psi=\psi_{0} \Delta \psi$.

\section{Helium atom}

The helium atom has both nuclear and electron singularities and therefore is a very interesting example. There are a lot of calculations in the literature with various types of functions, like Hylleraas [33], Kinosita [34], Pekeris [35], Thakkar and Koga [36], and others [37]. In particular, recently Schwartz [38] has reported very accurate calculations, giving the energy correct up to 35 decimal figures. Though these wave functions were cleverly formulated, the physics behind was not necessarily clear, except for the first few dominant terms. For general atomic and molecular systems, it is difficult to estimate the functional form of the exact wave function only by intuition as cleverly as in the heliumatom case. In the present ICI formalism, the wave function is automatically generated in an analytical form, once we choose the initial function $\psi_{0}$ and the scaling factor $g$, and it is guaranteed to approach the exact wave function. The purpose of the calculation presented here is to give a rough understanding of the free ICI method applied to helium atom: we start from a rather simple initial function $\psi_{0}$ using again a simple choice of the $g$ function. We examine what wave function the ICI method generates and how different it is from the existing wave functions. The state-of-the-art calculations of this atom with the ICI method will be published in a forthcoming paper [22].

The calculations are performed by using the coordinate defined by $s=r_{1}+r_{2}, t=-r_{1}+r_{2}$, and $u=r_{12}$ with the range of the variables $s \geqslant u \geqslant|t|$. With this coordinate, the Hamiltonian of the helium atom is written as

$$
\begin{aligned}
H= & -\frac{\partial^{2}}{\partial s^{2}}-\frac{\partial^{2}}{\partial t^{2}}-\frac{\partial^{2}}{\partial u^{2}}-2 \frac{s}{u} \frac{u^{2}-t^{2}}{s^{2}-t^{2}} \frac{\partial^{2}}{\partial s \partial u}-2 \frac{t}{u} \frac{u^{2}-s^{2}}{u t^{2}-s^{2}} \frac{\partial^{2}}{\partial u \partial t} \\
& -\frac{4 s}{s^{2}-t^{2}} \frac{\partial}{\partial s}-\frac{2}{u} \frac{\partial}{\partial u}-\frac{4 t}{t^{2}-s^{2}} \frac{\partial}{\partial t}-\frac{4 s Z}{s^{2}-t^{2}}+\frac{1}{u}
\end{aligned}
$$

where the last two terms represent the potential operator (nuclear attraction and electron repulsion) and the other terms represent the kinetic operator. The initial function we chose is a primitive one: $\psi_{0}=\exp (-\alpha s)$, with $\alpha$ being a variational parameter. The scaling function is $g=\left(V_{n e} V_{e e}\right)^{-1}$ $=u\left(s^{2}-t^{2}\right) / s$ to prevent the nuclear and electron singularity problem.

Table V summarizes the ground-state energy of the helium atom calculated with the free ICI method. As the iteration proceeds, the number of the independent functions generated by the free ICI method increases and the Ritz energy approaches the best variational value, -2.903724377 a.u., reported in the literature [38]. The chemical accuracy was 
TABLE V. Free ICI calculation of the helium atom with $\psi_{0}$ and $g$ given in the text.

\begin{tabular}{cccc}
\hline \hline Iteration $n$ & $M_{n}{ }^{\mathrm{a}}$ & Ritz energy & Optimal $\alpha$ \\
\hline 0 & 1 & -2.847656250 & 1.6875 \\
1 & 6 & -2.901577012 & 1.6728 \\
2 & 26 & -2.903708675 & 1.8803 \\
3 & 74 & -2.903723901 & 2.0330 \\
4 & 159 & -2.903724347 & 2.1998 \\
5 & 291 & -2.903724373 & 2.3307 \\
6 & 481 & -2.903724376 & 2.4862 \\
7 & 738 & -2.903724377 & $(2.68)$ \\
Best value $^{\mathrm{b}}$ & & -2.903724377 & \\
\hline
\end{tabular}

${ }^{\mathrm{a}}$ Number of independent functions.

${ }^{\mathrm{b}}$ Reference [38].

obtained already at the second iteration with 26 functions (the energy is correct to four decimal figures). At the fourth iteration, the free ICI energy is correct to seven decimal figures and at the seventh iteration it is correct to nine decimal figures. Clearly, by continuing the iteration, we should be able to improve our wave function to any order. This is certainly the behavior we expected from the proof of the theory. We particularly want to note that these results were obtained automatically by the free ICI algorithm described above.

The present helium wave function generated automatically by the free ICI theory was different from those existing in the literature. It was the Hylleraas-type wave function written as

$$
\psi=\left(\sum_{l, m, n} c_{l m n} s^{l} t^{2 m} u^{n}\right) \exp (-\alpha s),
$$

but was different from the original Hylleraas wave function in that our $l$ ran both positive and negative regions, while the original $l$ was only positive as well as $m$ and $n$. Our wave function was closer to the Kinoshita one though different in higher-order terms. Actually, the functional form of the ICI wave function depends on the choices of the zeroth-order wave function $\psi_{0}$ and the $g$ factor. The Hylleraas expansion actually corresponds to choosing $g=u\left(s^{2}-t^{2}\right)$, differently from the present one. This implies that a different convergence would be obtained by a different choice of these functions. In this sense, the examinations of different functions in the description of the helium wave function given by Thakkar and Koga [36] are useful. More detailed examinations of the choice of $\psi_{0}$ and $g$ in the ICI formalism of the helium atom will be given in a forthcoming paper [22].

\section{CONCLUDING REMARKS}

In modern quantum chemistry, one first prepares some basis functions by empiricism, therefrom a set of orthonormalized orbitals (Hartree-Fock orbitals mostly), and then expands everything with these set of orbitals. The Hamiltonian is rewritten in a second-quantized form and the quantumchemical calculations are transformed into matrix and vector manipurations. Ultimately, the best theory is the full-CI method. However, due to the incompleteness of the basis functions first prepared, the full-CI solutions are usually far from the true analytical solutions of the SE. When we enlarge the basis function to make it closer to be complete, the full-CI dimension increases formidably and, therefore, this route of accurate quantum chemistry must be reconsidered.

We have reported previously [6-11] the methodologies to reduce the number of variables that are necessary to obtain the full-CI solutions into general singles and doubles (GSD) number [6] and even to unity [7] and showed that their actual performances were certainly good $[10,11]$. However, even in these theories, we had to handle full-CI number of configurations and this made these methods still not very useful.

In the previous communication [13], we proposed a general method of solving the Schrödinger equation in an analytical expansion form and the details of the proposed method are presented in this paper. The method has already been extended to solve the relativistic Dirac and DiracCoulomb equations in an analytical form and preliminary calculations were encouraging [23]. In these methodologies, the strategy is very different from that of the modern quantum chemistry described above. We first prepare an initial function $\psi_{0}$ and choose an appropriate $g$ function. Then, the free ICI method automatically generates the most appropriate basis functions (complement functions) for the system by using the Hamiltonian of the system. The Hamiltonian includes all the information of the system. Further, the theorem of the ICI guarntees that the resultant wave function has the structure of the exact wave function as the iteration proceeds. For efficient calculations of this step, we need a symbolic manipulation mathematical program like MAPLE and MATHEMATICA. This point is quite different from traditional modern quantum-chemistry methodologies. Finally, the unknown variables included in the free ICI wave function are determined by the variational principle or by some variants thereof.

The good performance of the calculations reported here and carried out in the author's laboratory [13,21-23] implies a high potentiality of the proposed new methodology. The present ICI formalism based on the SSE may become a basis of the general systematic method of calculating the exact wave functions of atoms and molecules in an analytical expansion form. It was remarkable that the present ICI wave functions were different from the existing ones. Hylleraas [33], James-Coolidge [39], Kolos [40], and others were clever enough to imagine the exact wave functions of helium and hydrogen molecule only with intuitions. But for general molecules, it is difficult to estimate the analytical form of the exact wave function only by intuition, but the present theory makes it possible to generate it automatically, since the differentiations (involved in the Hamiltonian) are always possible and, furthermore, the integrals in the secular equation step have no singularity problem. It is guaranteed that at convergence we will get the exact wave function.

A different description of the wave function is obtained, depending on the different choice of the initial function $\psi_{0}$ and the $g$ function. Since the exact wave function is a unique reality, one may feel this to be strange. But this is something like to using different complete functional sets for describing 
one entity. Many possibilities exist, but all the descriptions are on a single reality: the exact wave function. So one description may be more efficient than the other. Actually, we will show in the succeeding paper that different choices of $\psi_{0}$ and $g$ give finally the same result, but the convergence speeds are different. It is impossible to write up the exact wave function of many-electron atoms and molecules by a single analytical function (without including operators) except for one-particle cases, so that the best analytical expression would be in an analytical expansion form.

Since applying the Hamiltonian to some function means a series of differentiations and multiplications, it is always possible to obtain the free ICI wave function in the form of Eq. (31). Further, at convergence, this wave function is guaranteed to have the structure of the exact wave function: when the variables $\left\{c_{k, n}\right\}$ are determined by the variational principle, it gives the exact wave function. In our theory, the process of forming the functions given by Eq. (31) is rather easy when a symbolic mathematical program like MAPLE is used. Usually, the most time-consuming step is a step for the variational calculations of the coefficiens $\left\{c_{k, n}\right\}$. The best procedure is to solve the secular equation by calculating analytically the Hamiltonian and overlap integrals over the functions $\left\{\phi_{k}\right\}^{(n)}$. We used this method in the present and succeeding papers. However, different from differentiations, integrations are not always possible for any functions. This gives some restrictions on the choices of the $g$ function and $\psi_{0}$. For general molecular systems, this problem becomes more serious and we have to introduce different procedures. Though Slater-type functions were used in our former calculations, Gaussian-type functions would also be useful for $\psi_{0}$. The Hamiltonian operator and the $g$ function do not change the functional form so that the types of functions are determined on the choice of $\psi_{0}$. The integrations over the Gaussian-type functions are easier than for the Slater-type functions. This merit may make up the well-known deficiencies of the Gaussian type functions on the cusp properties. In principle, the cusp properties are improved even with the Gaussians when many (infinite) Gaussians are used. Another general procedure may be provided by the Monte Carlo-type approach [41]. A combination of the local energy procedure and the Monte Carlo sampling method may provide a useful tool. We note that our ICI wave functions should potentially give very good local energies, so that we can expect small mean-square deviations (variances) in the Monte Carlo integration processes. This is a key feature of the ICI wave function. Studies along these lines are now in progress in our laboratory.

Though we have concentrated in this paper only on the ground state, the present theory is applicable also to calculations of the excited states. They are calculated as higher solutions of the same secular equation. For efficient calculations, some considerations of the choice of initial function $\psi_{0}$ are necessary. For example, we may take a linear combination of several functions having the exponents covering both ground and excited states under study.

We have recently extended the present methodology to the relativistic case [23]. We have developed a general theory of analytically solving the exact solutions of the Dirac and Dirac-Coulomb equations. There, the ICI formalism was similar to the present nonrelativistic case. A new problem was variational collapse, which commonly occurs in the relativistic variational calculations. However, the exactness of the ICI formalism has shed light on this problem and an interesting reader may refer to Ref. [23].

In separate papers $[21,22,30]$, we are going to summarize the results of the applications of the present theory to the many-electron atoms $\mathrm{He}, \mathrm{Li}, \mathrm{Be}$, and $\mathrm{B}$ and to two-electron molecules $\mathrm{H}_{2}$ and $\mathrm{He}-\mathrm{H}^{+}$. We are also going to summarize the results for Hooke's atom in a more general context than that given in this paper.

\section{ACKNOWLEDGMENTS}

The author thanks H. Nakashima and T. Kurokawa for some helpful discussions. This research was supported by a grant for Creative Scientific Research from the Ministry of Education, Science, Culture, and Sports of Japan.
[1] P. A. M. Dirac, Proc. R. Soc. London, Ser. A 123, 714 (1929).

[2] K. Fukui, Theory of Orientation and Stereoselection (SpringerVerlag, Heidelberg, 1070); Acc. Chem. Res. 4, 57 (1971).

[3] R. B. Woodward, and R Hoffmann, Angew. Chem., Int. Ed. Engl. 8, 781 (1969).

[4] P. J. Knowles and N. C. Handy, Chem. Phys. Lett. 111, 315 (1984).

[5] G. L. Bendazzoli and S. Evangelisti, J. Chem. Phys. 98, 3141 (1993).

[6] H. Nakatsuji, J. Chem. Phys. 113, 2949 (2000).

[7] H. Nakatsuji and E. R. Davidson, J. Chem. Phys. 115, 2000 (2001).

[8] H. Nakatsuji, J. Chem. Phys. 115, 2465 (2001).

[9] H. Nakatsuji, J. Chem. Phys. 116, 1811 (2002).

[10] H. Nakatsuji and M. Ehara, J. Chem. Phys. 117, 9 (2002).

[11] H. Nakatsuji and M. Ehara, J. Chem. Phys. 122, 194108
(2005).

[12] H. Nakatsuji, Phys. Rev. A 65, 052122 (2002).

[13] H. Nakatsuji, Phys. Rev. Lett. 93, 030403 (2004).

[14] M. Nooijen, Phys. Rev. Lett. 84, 2108 (2000).

[15] T. Van Voorhis and M. Head-Gordon, J. Chem. Phys. 115, 5033 (2001).

[16] P. Piecuch, K. Kowalski, P.-D. Fan, and K. Jedziniak, Phys. Rev. Lett. 90, 113001 (2003).

[17] E. R. Davidson, Phys. Rev. Lett. 91, 123001 (2003).

[18] S. Ronen, Phys. Rev. Lett. 91, 123002 (2003).

[19] D. A. Mazziotti, Phys. Rev. A 69, 012507 (2004).

[20] D. Mukherjee and W. Kutzelnigg, Chem. Phys. Lett. 397, 174 (2004); W. Kutzelnigg and D. Mukherjee, Phys. Rev. A 71, 022502 (2005).

[21] Y. Kurokawa, H. Nakashima, and H. Nakatsuji, this issue, Phys. Rev. A 72, 062502 (2005). 
[22] H. Nakashima, Y. Kurokawa, and H Nakatsuji (unpublished).

[23] H. Nakatsuji and H. Nakashima, Phys. Rev. Lett. 95, 050407 (2005).

[24] R. Kosloff and H. Tal-Ezer, Chem. Phys. Lett. 127, 223 (1986).

[25] T. Kato, Commun. Pure Appl. Math. 10, 151 (1957).

[26] D. Horn and M. Weinstein, Phys. Rev. D 30, 1256 (1984).

[27] J. Cioslowski, Phys. Rev. Lett. 58, 83 (1987).

[28] H. Huang, Q. Xie, Z. Cao, Z. Li, Z. Yue, and L. Ming, J. Chem. Phys. 110, 3703 (1999).

[29] D. Andrae, Phys. Rep. 336, 413 (2000).

[30] H. Nakatsuji, Bull. Chem. Soc. Jpn. 78, 1705 (2005).

[31] N. R. Kestner and O. Sinanoglu, Phys. Rev. 128, 2687 (1962).

[32] M. Taut, Phys. Rev. A 48, 3561 (1993).

[33] E. A. Hylleraas, Z. Phys. 54, 347 (1929).
[34] T. Kinishita, Phys. Rev. 105, 1490 (1957).

[35] C. L. Pekeris, Phys. Rev. 112, 1649 (1958).

[36] A. J. Thakkar and T. Koga, Phys. Rev. A 50, 854 (1994).

[37] J. Rychlewski and J. Komasa, in Explicitly Correlated Wave Functions in Chemistry and Physics-Theory and Applications, edited by J. Rychlewski (Kluwer Academic, Dordrecht, 2003), pp. 91-147.

[38] C. Schwartz, http://arxiv.org/PS_cache/physics/pdf/0208/ 0208004.pdf

[39] H. M. James and A. S. Coolidge, J. Chem. Phys. 1, 825 (1933).

[40] W. Kolos, J. Chem. Phys. 101, 1330 (1994).

[41] B. L. Hammond, W. A. Lester, Jr., and P. J. Reynolds, Monte Carlo Methods in Ab Initio Quantum Chemistry (World Scientific, Singapore, 1994). 